\title{
Robust Control Of A Flexible Manipulator Arm: A Benchmark Problem
}

\author{
Stig Moberg, Jonas Öhr \\ Division of Automatic Control \\ Department of Electrical Engineering \\ Linköpings universitet, SE-581 83 Linköping, Sweden \\ WWW: http://www. control.isy.liu.se \\ E-mail: stig@isy.liu.se, jonas.f.ohr@se.abb.com
}

20th April 2006

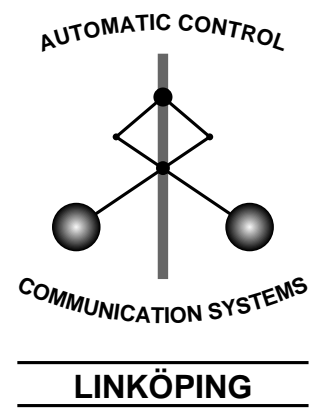

Report no.: LiTH-ISY-R-2685

Submitted to 16th IFAC World Congress, Prague, Czech Republic, July 4-8, 2005

Technical reports from the Control \& Communication group in Linköping are available at http://www. control.isy.liu.se/publications. 


\begin{abstract}
A control engineering benchmark problem with industrial relevance is presented. The process is a simulation model of a nonlinear four-mass system, which should be controlled by a discrete-time controller that optimizes performance for given robustness requirements. The control problem concerns only the so-called regulator problem.
\end{abstract}

Keywords: Robust control, benchmark examples, manipulators, disturbance rejection, flexible arms, QFT, sliding mode 


\title{
ROBUST CONTROL OF A FLEXIBLE MANIPULATOR ARM: A BENCHMARK PROBLEM
}

\author{
Stig Moberg* Jonas Öhr** \\ * ABB Automation Technologies AB - Robotics, \\ S-721 68 Västerås, Sweden \\ stig.moberg@se.abb.com \\ ** ABB AB - Corporate Research, \\ S-721 78 Västerås, Sweden \\ jonas.f.ohr@se.abb.com
}

\begin{abstract}
A control engineering benchmark problem with industrial relevance is presented. The process is a simulation model of a nonlinear four-mass system, which should be controlled by a discrete-time controller that optimizes performance for given robustness requirements. The control problem concerns only the so-called regulator problem. Copyright @ 2005 IFAC
\end{abstract}

Keywords: Robust control, benchmark examples, manipulators, disturbance rejection, flexible arms, QFT, sliding mode

\section{INTRODUCTION}

Researchers active within the academic systems and control society develop and propose very sophisticated and advanced methods for controller design and synthesis. However, some of the proposed methods and solutions have seldom been applied to advanced, realistic and industrially relevant problems. This is unfortunate for many reasons. For control engineers active in industry, e.g., it would be a great help to learn about the potentials (and maybe also the drawbacks) of a proposed method in the light of a realistic example. Some of the academic researchers could learn valuable lessons from the feedback that realistic experiments often give, lessons that might help pointing out new directions for future research.

This paper presents a realistic and relevant industrial benchmark problem and the intention is to stimulate research in the area of robust control of flexible industrial manipulators (robots). Proposed solutions to this benchmark problem will also be presented and discussed. An example of a similar (at least in some parts) benchmark problem is the flexible transmission system presented by (Landau et al., 1995). Another benchmark problem for controller design is (Graebe, 1994) where the participants did not know the true system, which was supplied in the form of scrambled simulation code. A third example is the Grumman F-14 Benchmark Control Problem described in (Rimer and Frederick, 1987). However, in the area of robot manipulator control we think that a realistic and relevant industrial benchmark problem is needed for reasons stated above.

\section{THE MANIPULATOR}

The most common type of industrial manipulator has six serially mounted links, all controlled by electrical motors via gears. Such a serial sixdegrees-of-freedom (6 DOF) industrial manipulator is shown in Figure 1. 
The dynamics of a serial 6 DOF manipulator change rapidly as the robot arms move fast within its working range and the dynamic couplings between the arms are strong. Moreover, the arm structure is elastic and the gears have nonlinearities in form of backlash, friction and nonlinear stiffness (elasticity).

From a control engineering perspective such a manipulator can be described as a nonlinear multivariable dynamical system having the six motor currents as the inputs and the six measurable motor angles as outputs. The goal of the motion control is, however, to control the orientation and the position $(3+3=6 \mathrm{DOF})$ of the tool when moving the tool along a certain desired path. It is a big challenge to control such an industrial manipulator so that high accuracy is guaranteed.

The benchmark problem described in this paper concerns only the so-called regulator problem and the controller should be designed such that the actual tool position is close to the desired reference, in the presence of motor torque disturbances, e.g. motor torque ripple, and tool disturbances acting on the tool e.g. under material processing. Since

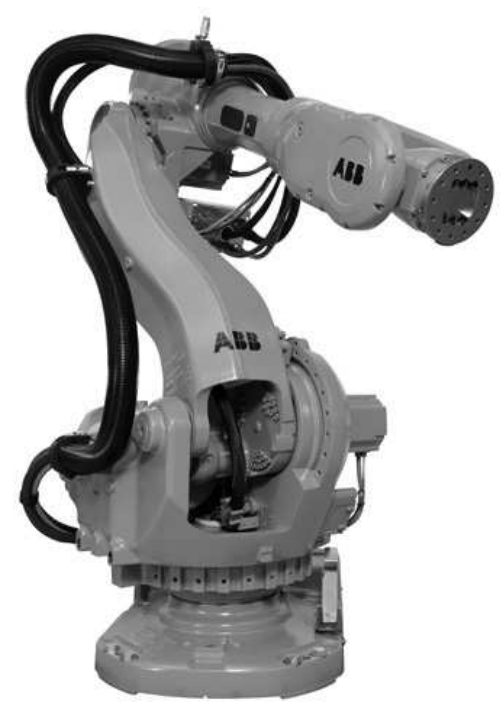

Fig. 1. IRB6600 from ABB

one direction of future manipulator development points toward increased elasticity, the motion control for high accuracy will require the use of more advanced control methods than already used today. Hence, the benchmark problem has great practical significance.

\section{THE SIMULATION MODEL}

The simulation model that will be used is a simplified SISO model and corresponds to one decoupled robot axis that operates close to a fixed operating point. The strong couplings between the six axes of the real manipulator, as well as the nonlinear dynamics associated with the change of configuration (operating point), will then be ignored. Furthermore, the nonlinear friction present in the gear is also ignored. The model to be used is a four-mass model having nonlinear gear elasticity and a time delay $T_{d}$ in the measurement system of the motor angular position. The motor current- and torque control is assumed to be ideal so that the motor torque becomes the model input. The model is illustrated in Figure 2.

The damping and the nonlinear stiffness of the gear are represented by $d_{1}$ and $k_{1}$ respectively and the corresponding parameters for the arm are represented by $d_{2}, k_{2}, d_{3}$ and $k_{3}$. The nonlinear stiffness of the gear is further described in section 5 and illustrated in Figure 3. The moment of inertia of the arm is here split-up in the three components $J_{a 1}, J_{a 2}$ and $J_{a 3}$. The moment of inertia of the motor is $J_{m}$. The parameters $f_{m}$, $f_{a 1}, f_{a 2}$ and $f_{a 3}$ represent viscous friction in the motor and in the arm structure respectively. $q_{m}$ is the motor angle which is measured. $q_{a 1}, q_{a 2}$ and $q_{a 3}$ are arm angles of the three masses and together they define the position of the tool. The angles in this model are, however, expressed on the high-speed side of the gear so in order to get the real arm angles one must divide the model angles by the gear-ratio (see the next section). The motor torque $u_{m}$, is the manipulated input of the system and $w$ and $v$ represent disturbances acting on the motor and the tool respectively.

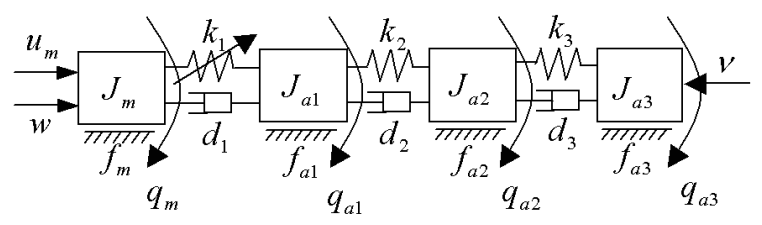

Fig. 2. Nonlinear model of the robot arm

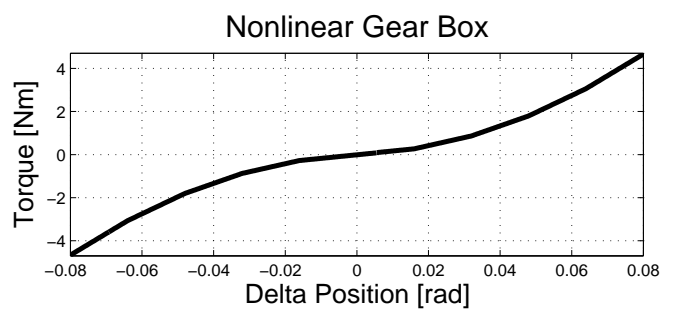

Fig. 3. Nonlinear gear stiffness

\section{MATHEMATICAL DESCRIPTION OF THE LINEARIZED MODEL}

The linearized model $\left(k_{1}=\right.$ constant, $\left.T_{d}=0\right)$ is given by the following equation:

$$
J \ddot{q}+(D+F) \dot{q}+K q=u
$$


where

$$
q=\left[\begin{array}{llll}
q_{m} & q_{a 1} & q_{a 2} & q_{a 3}
\end{array}\right]^{T}
$$

and

$$
u=\left[\begin{array}{c}
u_{m}+w \\
0 \\
0 \\
v
\end{array}\right]
$$

Furthermore

$$
\begin{gathered}
J=\left[\begin{array}{cccc}
J_{m} & 0 & 0 & 0 \\
0 & J_{a 1} & 0 & 0 \\
0 & 0 & J_{a 2} & 0 \\
0 & 0 & 0 & J_{a 3}
\end{array}\right] \\
D=\left[\begin{array}{cccc}
d_{1} & -d_{1} & 0 & 0 \\
-d_{1} & d_{1}+d_{2} & -d_{2} & 0 \\
0 & -d_{2} & d_{2}+d_{3} & -d_{3} \\
0 & 0 & -d_{3} & d_{3}
\end{array}\right] \\
F=\left[\begin{array}{cccc}
f_{m} & 0 & 0 & 0 \\
0 & f_{a 1} & 0 & 0 \\
0 & 0 & f_{a 2} & 0 \\
0 & 0 & 0 & f_{a 3}
\end{array}\right] \\
K=\left[\begin{array}{ccccc}
k_{1} \\
-k_{1} & k_{1}+k_{1} & 0 & 0 \\
0 & -k_{2} & k_{2}+k_{3} & -k_{3} \\
0 & 0 & -k_{3} & k_{3}
\end{array}\right]
\end{gathered}
$$

The tool position $z$ (which is the controlled variable) can for small variations around a given working point be calculated as

$$
z=\frac{l_{1} q_{a 1}+l_{2} q_{a 2}+l_{3} q_{a 3}}{n}
$$

where $n$ is the gear-ratio and $l_{1}, l_{2}, l_{3}$ are distances between the (fictive) masses and the tool.

On state-space form the linearized system can now be described by

$$
\begin{aligned}
\dot{x}(t) & =A x(t)+B u(t) \\
y(t) & =C x(t) \\
z(t) & =E x(t)
\end{aligned}
$$

where $y$ is the measured motor angle and $z$ the controlled variable. By selecting the states as

$$
x=\left[\begin{array}{c}
q \\
\dot{q}
\end{array}\right]
$$

we get

$$
\begin{aligned}
& A=\left[\begin{array}{cc}
0 & I \\
-J^{-1} K & -J^{-1}(D+F)
\end{array}\right] \\
& B=\left[\begin{array}{c}
0 \\
J^{-1}
\end{array}\right] \\
& C=\left[\begin{array}{llllllll}
1 & 0 & 0 & 0 & 0 & 0 & 0 & 0
\end{array}\right] \\
& E=\left[\begin{array}{llllllll}
0 & \frac{l_{1}}{n} & \frac{l_{2}}{n} & \frac{l_{3}}{n} & 0 & 0 & 0 & 0
\end{array}\right]
\end{aligned}
$$

\section{NOMINAL MODEL}

The parameter values of the nominal model, which will be denoted by $M_{\text {nom }}$, is defined in Table 1 .

The nonlinear gear stiffness illustrated in Figure 3 is approximated and described by five piecewise linear segments in the simulation model. In the table only the first segment, $k_{1, \text { low }}$, the last segment, $k_{1, \text { high }}$, and the position difference where the last segment begins, $k_{1, p o s}$, are given.

Table 1. Nominal parameter values

\begin{tabular}{lll}
\hline Parameter & Value & Unit \\
\hline$J_{m}$ & $5 \cdot 10^{-3}$ & $\mathrm{~kg} \cdot \mathrm{m}^{2}$ \\
$J_{a 1}$ & $2 \cdot 10^{-3}$ & $\mathrm{~kg} \cdot \mathrm{m}^{2}$ \\
$J_{a 2}$ & 0.02 & $\mathrm{~kg} \cdot \mathrm{m}^{2}$ \\
$J_{a 3}$ & 0.02 & $\mathrm{~kg} \cdot \mathrm{m}^{2}$ \\
$k_{1, \text { high }}$ & 100 & $\mathrm{Nm} / \mathrm{rad}$ \\
$k_{1, \text { low }}$ & 16.7 & $\mathrm{Nm} / \mathrm{rad}$ \\
$k_{1, \text { pos }}$ & 0.064 & $\mathrm{rad}$ \\
$k_{2}$ & 110 & $\mathrm{Nm} / \mathrm{rad}$ \\
$k_{3}$ & 80 & $\mathrm{Nm} / \mathrm{rad}$ \\
$d_{1}$ & 0.08 & $\mathrm{Nm} \cdot \mathrm{s} / \mathrm{rad}$ \\
$d_{2}$ & 0.06 & $\mathrm{Nm} \cdot \mathrm{s} / \mathrm{rad}$ \\
$d_{3}$ & 0.08 & $\mathrm{Nm} \cdot \mathrm{s} / \mathrm{rad}$ \\
$f_{m}$ & $6 \cdot 10^{-3}$ & $\mathrm{Nm} \cdot \mathrm{s} / \mathrm{rad}$ \\
$f_{a 1}$ & $1 \cdot 10^{-3}$ & $\mathrm{Nm} \cdot \mathrm{s} / \mathrm{rad}$ \\
$f_{a 2}$ & $1 \cdot 10^{-3}$ & $\mathrm{Nm} \cdot \mathrm{s} / \mathrm{rad}$ \\
$f_{a 3}$ & $1 \cdot 10^{-3}$ & $\mathrm{Nm} \cdot \mathrm{s} / \mathrm{rad}$ \\
$n$ & 220 & \\
$l_{1}$ & 20 & $\mathrm{~mm}$ \\
$l_{2}$ & 600 & $\mathrm{~mm}$ \\
$l_{3}$ & 1530 & $\mathrm{~mm}$ \\
$T_{d}$ & $0.5 \cdot 10^{-3}$ & $\mathrm{~s}$ \\
\hline & & \\
\end{tabular}

\section{PARAMETER VARIATIONS AND MODEL SETS}

Performance of the control systems will be evaluated for the nominal model $M_{\text {nom }}$ and for two sets of models which will be denoted by $M_{1}$ and $M_{2}$ respectively, and which contain ten models, $\mathrm{m}$, each. The set $M_{1}$ represents relatively small variations in the physical parameters and the set $M_{1}$ represents relatively large.

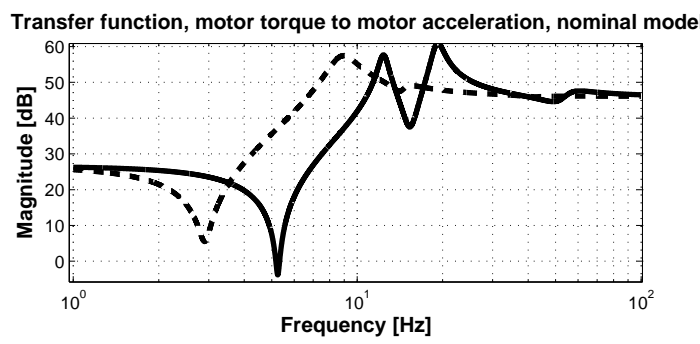

Fig. 4. Amplitude curves for $M_{\text {nom }}$

In Figure 4 the frequency response function amplitude for $\frac{\ddot{Q}(\omega)}{U(\omega)}$ of $M_{n o m}$ is shown. The solid line corresponds to the most stiff region of the gear $\left(k_{1, \text { high }}\right)$ and the dashed line corresponds to the least stiff region $\left(k_{1, \text { low }}\right)$. The Figures 5 and 6 show 
the corresponding curves of the models $m \in M_{1}$ and $m \in M_{2}$ respectively, for the most stiff region of the gear $\left(k_{1, \text { high }}\right)$. 1

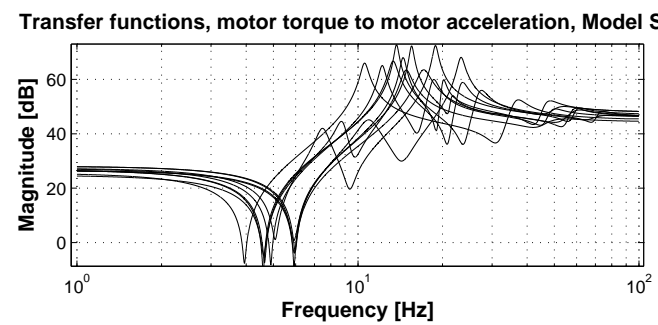

Fig. 5. Amplitude curves $\forall m \in M_{1}$

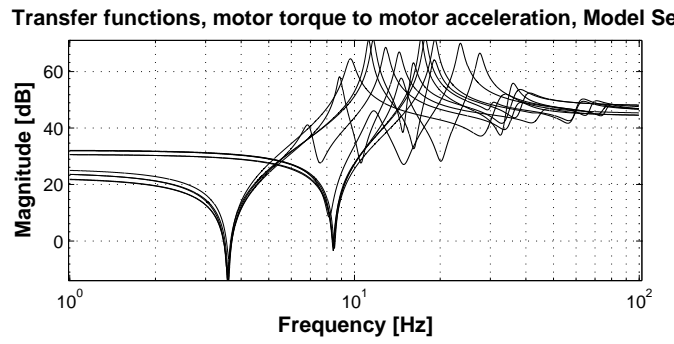

Fig. 6. Amplitude curves $\forall m \in M_{2}$

\section{THE TASK}

The task of this benchmark problem is to design one or two discrete time controllers for the systems described above. One of the controllers must be capable of controlling all the models $m \in M_{n o m} \cup M_{1} \cup M_{2}$ whereas the other controller should be able to control $M_{\text {nom }}$ alone. The controllers can be linear or nonlinear.

In order to investigate how well a controller can perform when really good models are available it is recommended to design two different controllers where one is optimized for the control of $M_{n o m}$ only. This controller will in the sequel be denoted by $C_{1}$ and the other by $C_{2}$. Note that $C_{1}$ and $C_{2}$ can be identical, can have the same structure and differ only by different tuning or can have completely different structure and tuning parameters.

The control systems will be exposed to a sequence of torque disturbances acting on the motor and on the tool according to Figure 7. The disturbance sequence consists of steps, pulses and sweeping sinusoids (chirp). Figure 8 shows the tool position and Figure 9 shows the controller signal (motor torque) when the disturbance sequence in Figure 7 acts on the nominal system. A simple PID-type controller is used here.

As a tool for controller design, a cost function will be used. The smaller the cost function the

\footnotetext{
1 In the simulation model, $M_{1}$ and $M_{2}$ also have the nonlinear gear stiffness described in section 5 .
}

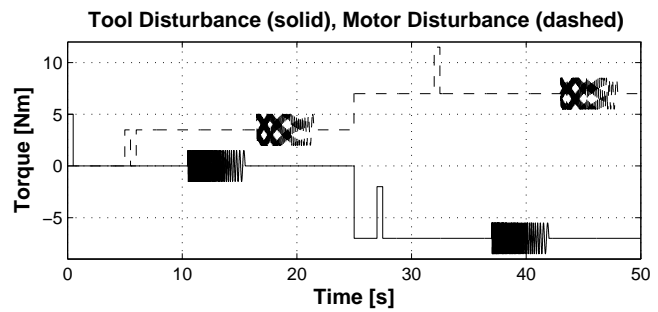

Fig. 7. Torque disturbances on motor and tool

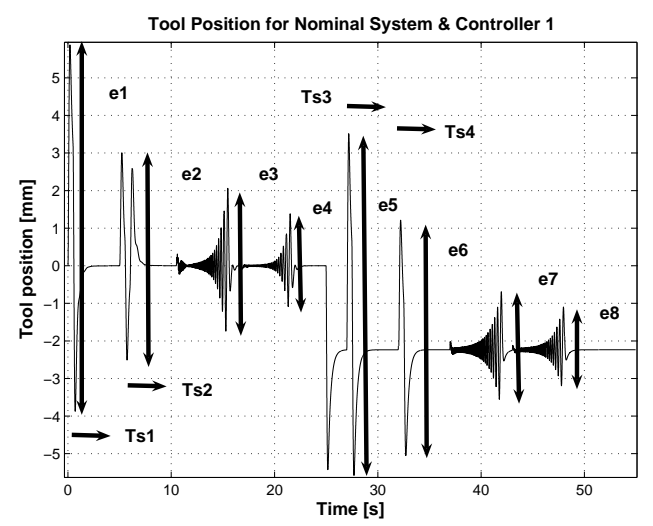

Fig. 8. Tool position when using PID-control

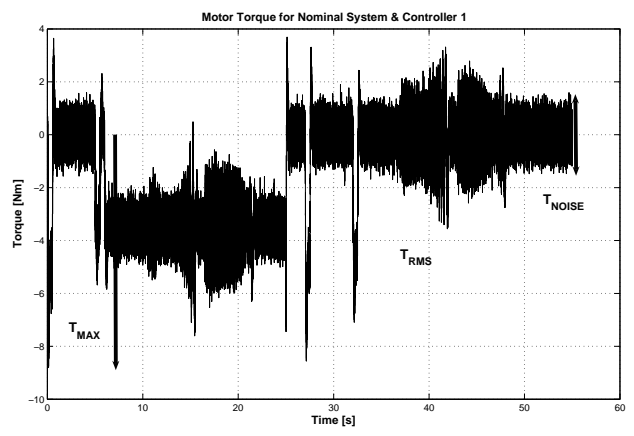

Fig. 9. Motor torque when using PID-control

better the design. Figures 8 and 9 show all the performance measures that are weighted together into this cost function. Peak-to-peak error of the tool position $\left(e_{1}-e_{8}\right)$, settling times $\left(T s_{1}-T s_{4}\right)$, maximum torque $T_{M A X}$, adjusted rms torque $T_{R M S}$ and torque "noise" (peak-to-peak) $T_{\text {NOISE }}$.

Note that $T_{\text {NOISE }}$, which can be measurement noise and/or chattering caused by a discontinuous controller, is measured by the simulation routines when the system is at rest but that a good controller would keep the chattering/noise on a decent level also when it operates actively.

For the nominal system the following cost function $V_{\text {nom }}$ will be used:

$$
V_{n o m}=\gamma \sum_{i=1}^{15} \alpha_{i} e_{i}
$$

where $e_{i}$ represents a generalized "error" (position error, settling time or torque), $\gamma$ and $\alpha_{i}$ are 
weights. It should be mentioned that $V_{\text {nom }}$ is calculated only when $C_{1}$ is used for the control of $M_{\text {nom }}$. For the sets $M_{1}$ and $M_{2}$ (using controller $C_{2}$ ) the maximum error from the simulations is used and the cost functions $V_{1}$ and $V_{2}$ are given by the following expressions:

$$
\begin{aligned}
& V_{1}=\gamma \sum_{i=1}^{15} \alpha_{i} \max _{m \in M_{1}}\left(e_{i}\right) \\
& V_{2}=\gamma \sum_{i=1}^{15} \alpha_{i} \max _{m \in M_{2}}\left(e_{i}\right)
\end{aligned}
$$

The total cost function $V$ is given by

$$
V=\beta_{\text {nom }} V_{\text {nom }}+\beta_{1} V_{1}+\beta_{2} V_{2}
$$

The following requirements must also be met:

- Settling times for $M_{n o m}\left(C_{1}\right)$ and $M_{1}\left(C_{2}\right)$ : $T s_{1,2,3,4}<3 \mathrm{~s}$, error band $\pm 0.1 \mathrm{~mm}$

- Settling times for $M_{2}\left(C_{2}\right): T s_{1,2,3,4}<4 \mathrm{~s}$, error band $\pm 0.3 \mathrm{~mm}$

- Torque noise $T_{N O I S E}<5 \mathrm{Nm}$ for all systems

- Stability for all systems

- Stability for $M_{\text {nom }}$ when increasing the loop gain by a factor 2.5 for $C_{1}$ and $C_{2}$

- Stability for $M_{\text {nom }}$ when increasing the delay $T_{d}$ from $0.5 \mathrm{~ms}$ to $2 \mathrm{~ms}$ for $C_{1}$ and $C_{2}$

By "stability" we also mean that limit cycles larger than $10 \mu \mathrm{m}$ peak-to-peak are not allowed.

The control requirements we put on the systems $M_{n o m}$ and $M_{1}$ above can be motivated by e.g. differences between robot individuals, non modeled nonlinearities, model reduction, uncertainties due to varying applications and incorrect payload definition. The use of the model set $M_{2}$ is motivated by the fact that a real control system must be stable even for relatively large deviations between the model and the real manipulator dynamics. It might then seem strange to weight performance for the set $M_{2}$ into the total cost function but the reason is that we want to reward the robustness of the proposed controller.

\section{THE CONTROL SYSTEM}

The control system is shown in form of a Simulink ${ }^{T M}$-model in Figure 10. The following conditions rules:

- Sampling time $0.5 \mathrm{~ms}$

- Time delay $T_{d} 0.5 \mathrm{~ms}$

- Torque saturation limits $\pm 20 \mathrm{Nm}$ (the saturation function in the controller block should not be removed)

- Relatively strong measurement noise (only the motor shaft angle is measured)

- Only the blocks "Controller1" and "Controller2" and the files "Controller_1.m" and

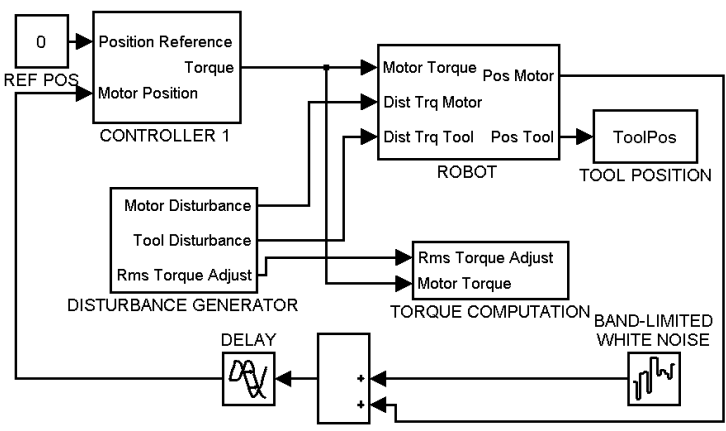

Fig. 10. The control system

"Controller_2.m" are allowed to be changed.

The system comes with a simple PID-controller

- No continuous-time blocks can be added

- No knowledge of deterministic nature about the noise and disturbances can be used in the controller (of course)

- The controller $C_{2}$ must have the same initial states and parameter values for all the simulations of $m \in M_{\text {nom }} \cup M_{1} \cup M_{2}$

The control system and the models are described in detail by Matlab ${ }^{\mathrm{TM}}$ and Simulink ${ }^{\mathrm{TM}}$ files available for download at http://www.robustcontrol.org. The Matlab ${ }^{\mathrm{TM}}$ products used are described in e.g. (MathWorks, 2003).

\section{SUGGESTED SOLUTIONS}

This benchmark problem was first presented as "Swedish Open Championships in Robot Control" at the Swedish control meeting, Chalmers University of Technology, Sweden (Moberg and Öhr, 2004). On request, this competition was spread outside the borders of Sweden.

Three interesting solutions for the competition were proposed:

- QFT controller by P.-O. Gutman, Israel Institute of Technology, Israel

- Linear Sliding Mode Controller by W.-H. Zhu, Canadian Space Agency, Canada

- Polynomial Controller by F. Sikström and A.-K. Christiansson, University of Trollhättan/Uddevalla, Sweden

The controllers are of order 3 to 7 . The QFT approach is generally described in (Nordin and Gutman, 1995) and the linear sliding mode approach in (Zhu et al., 1992) and (Zhu et al., 2001). The polynomial controller is optimized for the given cost function and the optimization procedures used are described in (MathWorks, 1999). ${ }^{2} 3$

\footnotetext{
2 The winner of the championship was W.-H. Zhu, Canadian Space Agency, Canada.

3 A fuzzy PID controller for the nominal case only was proposed by J. Varso and H. Koivo, Helsinki University of Technology. $V_{\text {nom }}$ for this controller is 59.3
} 
A second QFT controller of order 13 was proposed in the Master Thesis Work described in (Roberto, 2005) but since the authors of this article supervised that work, the solution did not qualify for the competition.

The frequency response of the controllers are shown in Figure 11 and 12. The cost function

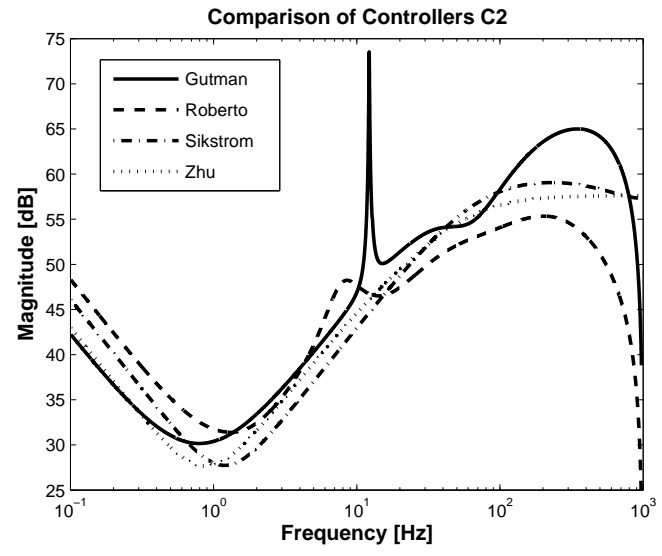

Fig. 11. The Frequency Response Amplitude of the Controllers $C_{2}$

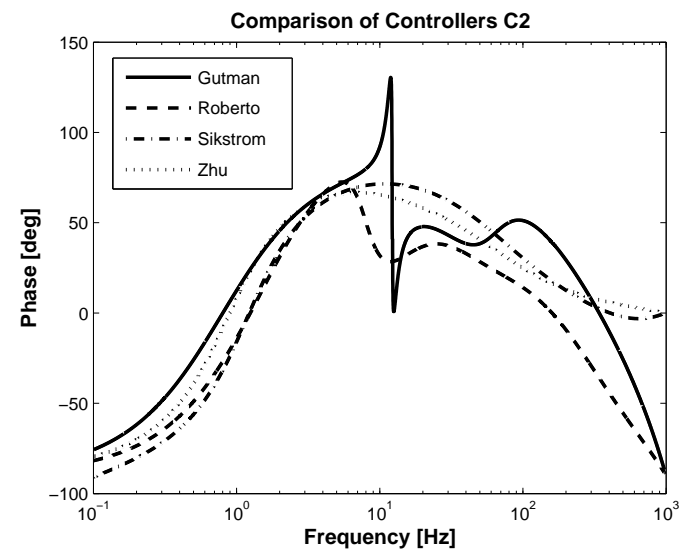

Fig. 12. The Frequency Response Phase of the Controllers $C_{2}$

$V_{1}$ and the generalized errors for model set $M_{1}$ are shown in Table 2. Table 3 shows a summary of the result.

Since a real manipulator has multiple inputs and outputs, a second step in the evolution of this benchmark problem could be to look at the multivariable case and/or using additional measured variables like e.g. arm position or tool position.

\section{ACKNOWLEDGMENTS}

The authors would like to thank those who have contributed with solutions to the benchmark problem. Valuable help and support have gratefully been received from Torgny Brogårdh, Staffan Elfving, Svante Gunnarsson and Alf Isaksson.
Table 2. Result for $M_{1}$

\begin{tabular}{lllll}
\hline & Gutman & Roberto & Sikström & Zhu \\
\hline$e_{1}[\mathrm{~mm}]$ & 8.22 & 8.57 & 9.75 & 9.11 \\
$e_{2}[\mathrm{~mm}]$ & 2.56 & 2.43 & 3.41 & 3.22 \\
$e_{3}[\mathrm{~mm}]$ & 5.39 & 5.56 & 5.34 & 5.28 \\
$e_{4}[\mathrm{~mm}]$ & 1.58 & 1.74 & 2.12 & 1.77 \\
$e_{5}[\mathrm{~mm}]$ & 7.78 & 8.22 & 9.37 & 8.64 \\
$e_{6}[\mathrm{~mm}]$ & 2.82 & 2.82 & 4.02 & 3.68 \\
$e_{7}[\mathrm{~mm}]$ & 4.88 & 5.13 & 4.20 & 4.59 \\
$e_{8}[\mathrm{~mm}]$ & 1.40 & 1.56 & 1.90 & 1.57 \\
$T s_{1}[\mathrm{~s}]$ & 2.04 & 2.13 & 1.79 & 1.68 \\
$T s_{2}[\mathrm{~s}]$ & 1.25 & 1.47 & 1.52 & 1.05 \\
$T s_{3}[\mathrm{~s}]$ & 1.04 & 0.77 & 0.71 & 0.77 \\
$T s_{4}[\mathrm{~s}]$ & 0.95 & 0.55 & 0.69 & 0.71 \\
$T_{N O I S E}[\mathrm{Nm}]$ & 2.67 & 1.05 & 1.85 & 1.66 \\
$T_{M A X}[\mathrm{Nm}]$ & 12.1 & 12.0 & 11.0 & 11.3 \\
$T_{R M S}[\mathrm{Nm}]$ & 1.53 & 1.52 & 1.43 & 1.46 \\
\hline$V_{1}$ & 82.5 & 80.8 & 84.8 & 80.5 \\
\hline
\end{tabular}

Table 3. Summary of Result

\begin{tabular}{lllll}
\hline & Gutman & Roberto & Sikström & Zhu \\
\hline$V_{\text {nom }}$ & 64.6 & 58.8 & 64.8 & 62.0 \\
$V_{1}$ & 82.5 & 80.8 & 84.8 & 80.5 \\
$V_{2}$ & 82.6 & 84.2 & 84.1 & 81.6 \\
\hline$V$ & 146.0 & 141.4 & 148.9 & 142.2 \\
\hline
\end{tabular}

\section{REFERENCES}

Graebe, S.F. (1994). Robust and adaptive control of an unknown plant: A benchmark of new format. Automatica 30(4), 567-575.

Landau, I.D., D. Ray, A. Karimi, A. Voda and A. Franco (1995). A flexible transmission system as a benchmark for robust digital control. European Journal of Control 1(2), 77-96.

MathWorks (1999). MATLAB Optimization Toolbox Users Guide. The MathWorks Inc.. Natic, Mass.

MathWorks (2003). MATLAB and Simulink Users Guide. The MathWorks Inc.. Natic, Massachusetts, USA.

Moberg, S. and J. Öhr (2004). Svenskt mästerskap i robotreglering. In: Swedish Control Meeting 2004, Göteborg, Sweden.

Nordin, M. and P.-O. Gutman (1995). Digital QFT design for the benchmark problem. European Journal of Control 1(2), 97-103.

Rimer, M. and D.K. Frederick (1987). Solutions of the Grumman F-14 benchmark control problem. IEEE Control Systems Magazine $7(4), 36-40$.

Roberto, O. (2005). Robust control of industrial robot - development of QFT based design. Master's thesis. Uppsala University. Signals $\&$ Systems.

Zhu, W.-H., H.-T. Chen and Z.-J. Zhang (1992). A variable structure robot control algorithm with an observer. IEEE Transactions on Robotics and Automation 8(4), 486-492.

Zhu, W.-H., M.B. Yun and Y. Altintas (2001). A fast tool servo design for precision turning of shafts on conventional CNC lathes. International Journal of Machine Tools $\& 5$ Manufacture 41(7), 953-965. 


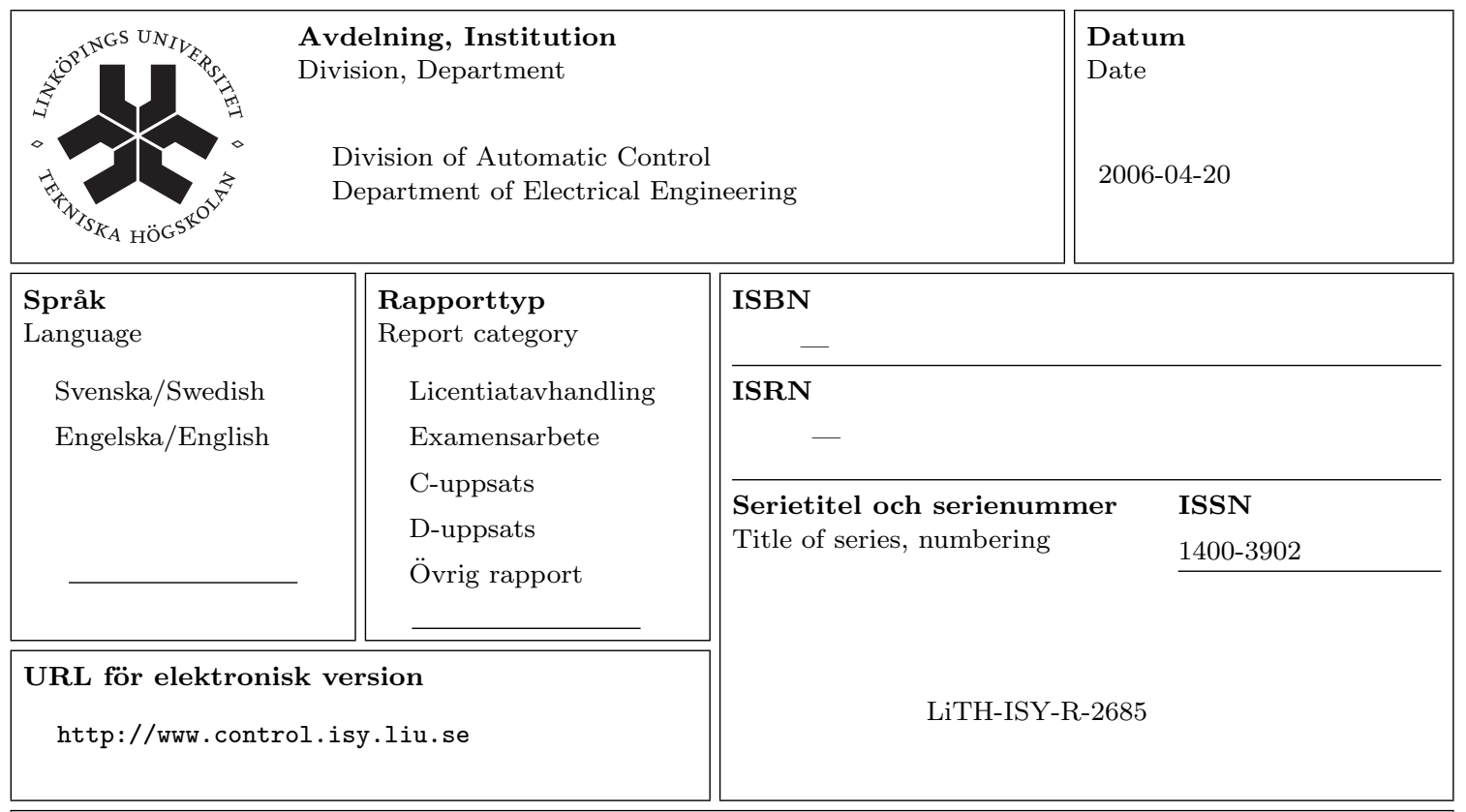

Titel Robust Control Of A Flexible Manipulator Arm: A Benchmark Problem

Title

Author

Sammanfattning

Abstract

A control engineering benchmark problem with industrial relevance is presented. The process is a simulation model of a nonlinear four-mass system, which should be controlled by a discrete-time controller that optimizes performance for given robustness requirements. The control problem concerns only the so-called regulator problem. 